\title{
Distance dependence of fluorescence resonance energy transfer ${ }^{\dagger}$
}

\author{
R S SWATHI and K L SEBASTIAN* \\ Department of Inorganic and Physical Chemistry, Indian Institute of Science, Bangalore 560012 \\ e-mail: kls@ipc.iisc.ernet.in
}

\begin{abstract}
Deviations from the usual $R^{-6}$ dependence of the rate of fluorescence resonance energy transfer (FRET) on the distance between the donor and the acceptor have been a common scenario in the recent times. In this paper, we present a critical analysis of the distance dependence of FRET, and try to illustrate the non- $R^{-6}$ type behaviour of the rate for the case of transfer from a localized electronic excitation on the donor, a dye molecule to three different energy acceptors with delocalized electronic excitations namely, graphene, a two-dimensional semiconducting sheet and the case of such a semiconducting sheet rolled to obtain a nanotube. We use simple analytic models to understand the distance dependence in each case.
\end{abstract}

Keywords. FRET; graphene; nanotube; tight-binding model.

\section{Introduction}

Resonance energy transfer is the process of nonradiative transfer of energy from an excited state donor to a ground state acceptor. The resonance condition implies that the emission spectrum of the donor has to have significant overlap with the absorption spectrum of the acceptor. ${ }^{1}$ When the donor is a fluorescent species, the process is called fluorescence resonance energy transfer (FRET). Acceptor may or may not be fluorescent. In FRET, the rate of energy transfer follows an $R^{-6}$ dependence where $R$ is the distance between the donor and the acceptor. The rate of energy transfer can be evaluated using the Fermi golden rule of time-dependent quantum mechanics: ${ }^{2}$

$$
\begin{gathered}
k=\frac{2 \pi}{\hbar} \sum_{i, f} \rho_{e v_{e}}^{D} \rho_{g u_{g}}^{A}\left|\left\langle\Psi_{e v_{e}}^{D} \Psi_{g u_{g}}^{A}\left|H_{I}\right| \Psi_{g v_{g}}^{D} \Psi_{e u_{e}}^{A}\right\rangle\right|^{2} \\
\delta\left(E_{e v_{e}}^{D}+E_{g u_{g}}^{A}-E_{g v_{g}}^{D}-E_{e u_{e}}^{A}\right) .
\end{gathered}
$$

$D$ and $A$ refer to the donor and the acceptor respectively. The initial state of the donor is an excited state denoted as $\Psi_{e v_{e}}^{D}$, with an energy $E_{e v_{e}}^{D}$. The donor is initially in an excited state with an electron that was sitting in the molecular orbital $\psi_{g}^{D}$ promoted to the orbital $\psi_{e}^{D}$. Initially, the acceptor is in the ground state, and after the energy transfer, an

${ }^{\dagger}$ Dedicated to the memory of the late Professor S K Rangarajan *For correspondence electron which was sitting in the molecular orbital $\psi_{g}^{A}$ is promoted to $\psi_{e}^{A}$. In the above process, there are changes in the vibrational states of both the donor and the acceptor. The vibrational quanta are denoted by $v_{e}, v_{g}, u_{g}$ and $u_{e} . \rho_{e v_{e}}^{D}$ and $\rho_{g u}^{A}$ are the initial distributions of the donor in the excited state and the acceptor in the ground state respectively. $H_{1}$ is the part of the Hamiltonian responsible for the energy transfer and is Coulombic in nature, as no other interaction is possible if the overlap between the orbitals of the donor and the acceptor is small (which is usually the case).

Within the adiabatic approximation, the matrix element may then be written as ${ }^{3}$

$$
\begin{aligned}
& \left\langle\Psi_{e v_{e}}^{D} \Psi_{g u_{g}}^{A}\left|H_{\mathrm{I}}\right| \Psi_{g v_{g}}^{D} \Psi_{e u_{e}}^{A}\right\rangle \\
= & \left\langle v_{e} u_{g}\left|\left\langle\psi_{e}^{D}(1) \psi_{g}^{A}(2)\left|\frac{e^{2}}{4 \pi \varepsilon r_{12}}\right| \psi_{g}^{D}(1) \psi_{e}^{A}(2)\right\rangle\right| v_{g} u_{e}\right\rangle,
\end{aligned}
$$

where $\varepsilon$ is the permittivity of the medium separating the donor and the acceptor. As a result of the Coulombic interaction, an electron in the donor gets de-excited, with the simultaneous excitation of an electron of the acceptor. Neglecting the nuclear position dependence of the matrix element

$$
\left\langle\psi_{e}^{D}(1) \psi_{g}^{A}(2)\left|\frac{e^{2}}{4 \pi \varepsilon r_{12}}\right| \psi_{g}^{D}(1) \psi_{e}^{A}(2)\right\rangle
$$


and approximating it by its value evaluated at the equilibrium positions of the nuclei as

$$
\begin{aligned}
& \left\langle\psi_{e}^{D}(1) \psi_{g}^{A}(2)\left|\frac{e^{2}}{4 \pi \varepsilon r_{12}}\right| \psi_{g}^{D}(1) \psi_{e}^{A}(2)\right\rangle \\
& \simeq\left\langle\psi_{e}^{D}(1) \psi_{g}^{A}(2)\left|\frac{e^{2}}{4 \pi \varepsilon r_{12}}\right| \psi_{g}^{D}(1) \psi_{e}^{A}(2)\right\rangle_{e q}
\end{aligned}
$$

leads to

$$
\begin{aligned}
& \left\langle\Psi_{e v_{e}}^{D} \Psi_{g u_{g}}^{A}\left|H_{\mathrm{I}}\right| \Psi_{g v_{g}}^{D} \Psi_{e u_{e}}^{A}\right\rangle \\
= & \left\langle\psi_{e}^{D}(1) \psi_{g}^{A}(2)\left|\frac{e^{2}}{4 \pi \varepsilon r_{12}}\right| \psi_{g}^{D}(1) \psi_{e}^{A}(2)\right\rangle_{e q}\left\langle v_{e} \mid v_{g}\right\rangle\left\langle u_{g} \mid u_{e}\right\rangle .
\end{aligned}
$$

Note that the integral

$$
\left\langle\psi_{e}^{D}(1) \psi_{g}^{A}(2)\left|\frac{e^{2}}{4 \pi \varepsilon r_{12}}\right| \psi_{g}^{D}(1) \psi_{e}^{A}(2)\right\rangle_{e q}
$$

(we shall from now onwards neglect the subscript $e q$ ) is just the electrostatic interaction between the transition densities $\psi_{e}^{D *}(1) \psi_{g}^{D}(1)$ and $\psi_{g}^{A *}(2) \psi_{e}^{A}(2)$. For large interparticle separations, this interaction may be approximated as the interaction between the corresponding transition dipoles. ${ }^{2}$ This is known as the dipolar approximation. Within this approximation,

$$
\begin{array}{r}
\left\langle\psi_{e}^{D}(1) \psi_{g}^{A}(2)\left|\frac{e^{2}}{4 \pi \varepsilon r_{12}}\right| \psi_{g}^{D}(1) \psi_{e}^{A}(2)\right\rangle \\
=\frac{1}{4 \pi \varepsilon} \frac{\boldsymbol{\mu}_{e g}^{D} \boldsymbol{\mu}_{g e}^{A}-3 \boldsymbol{\mu}_{e g}^{D} \hat{\mathbf{R}} \hat{\mathbf{R}} \cdot \boldsymbol{\mu}_{g e}^{A}}{R^{3}},
\end{array}
$$

where $\boldsymbol{\mu}_{e g}^{D}, \quad \boldsymbol{\mu}_{g e}^{A}$ are the transition dipoles of the donor and the acceptor respectively and are defined by

$$
\boldsymbol{\mu}_{e g}^{D}=-e \int d \mathbf{r} \psi_{e}^{D *}(\mathbf{r}) \mathbf{r} \psi_{g}^{D}(\mathbf{r}),
$$

and

$$
\boldsymbol{\mu}_{g e}^{A}=-e \int d \mathbf{r} \psi_{g}^{A *}(\mathbf{r}) \mathbf{r} \psi_{e}^{A}(\mathbf{r}) .
$$

$\hat{\mathbf{R}}$ is the unit vector in the direction of the vector $\mathbf{R}$ connecting the centres of charge of the two species. Due to the dipolar approximation of (4), the rate of transfer has an $R^{-6}$ dependence. Förster ${ }^{3}$ was the first to analyse this process theoretically and he arrived at the following expression for the rotationally averaged rate:

$$
k=\frac{1}{\tau_{0}}\left(\frac{R_{0}}{R}\right)^{6}
$$

where $\tau_{0}$ is the lifetime of the donor in the absence of the acceptor and $R_{0}$ is the well-known Förster radius. ${ }^{4}$ Since the experimental demonstration of FRET as a useful spectroscopic ruler by Stryer and Haugland, ${ }^{5}$ it has been used extensively for understanding the conformational dynamics of biological molecules like proteins, RNA, etc. When the donor and the acceptor are two dye molecules, FRET is found to be effective in the range $10 \AA-$ $100 \AA$. Energy transfer provides an additional deexcitation pathway for the donor thereby leading to a decrease in the lifetime of the excited donor in presence of the acceptor compared to that in the absence of the acceptor. After about $100 \AA$, in FRET, the excited donor decays according to its natural lifetime, rather than by energy transfer.

When either the donor or the acceptor or both are extended systems with delocalized charge densities, breakdown of the dipolar approximation leads to deviations from the usual $R^{-6}$ dependence. In view of the recent studies leading to non- $R^{-6}$ dependencies of the rate of energy transfer, we analyse the process of energy transfer from a dye molecule, which has a localized excitation to three different acceptors with delocalized excitations: graphene, a two-dimensional semiconducting sheet and a long semiconducting nanotube. We present a critical analysis of the distance dependence of the rate in all the cases. The paper is organized as follows: in the second section, we present a general formalism for evaluating the rate for the case of transfer from a localized donor to delocalized acceptors. Third, fourth and fifth sections deal with transfer to graphene, a two-dimensional semiconducting sheet and a long semiconducting nanotube respectively. In the sixth section, we present a general discussion of the non- $R^{-6}$ behaviour of the rate for various systems. 
2. Resonance energy transfer from a localized donor to acceptors with delocalized electron densities

We consider the process of resonance energy transfer from a localized donor, namely a dye molecule to acceptors with delocalized charge densities. The matrix element for interaction is given by

$$
U=\frac{e^{2}}{4 \pi \varepsilon} \int d \mathbf{r}_{1} \int d \mathbf{r}_{2} \frac{\psi_{e}^{D *}\left(\mathbf{r}_{1}\right) \psi_{g}^{D}\left(\mathbf{r}_{1}\right) \psi_{g}^{A *}\left(\mathbf{r}_{2}\right) \psi_{e}^{A}\left(\mathbf{r}_{2}\right)}{\left|\mathbf{r}_{1}-\mathbf{r}_{2}\right|}
$$

The orbitals $\psi_{g}^{A}\left(\mathbf{r}_{2}\right)$ and $\psi_{e}^{A}\left(\mathbf{r}_{2}\right)$ on the acceptor are extended in space (in comparison with the distance of the donor from the acceptor). In such a situation, we can think of the interaction between the donor and the acceptor as that between the transition dipole of the donor, $\boldsymbol{\mu}_{e g}^{D}$ located at the center of charge given by

$$
\boldsymbol{\mu}_{e g}^{D}=-e \int d \mathbf{r}_{1} \psi_{e}^{D *}\left(\mathbf{r}_{1}\right) \mathbf{r}_{1} \psi_{g}^{D}\left(\mathbf{r}_{1}\right)
$$

and the transition charge density $-e \psi_{g}^{A *}\left(\mathbf{r}_{2}\right) \psi_{e}^{A}\left(\mathbf{r}_{2}\right)$ of the acceptor. Therefore, the matrix element for interaction becomes

$$
U=\boldsymbol{\mu}_{e g}^{D} \cdot \nabla \Phi,
$$

where $\Phi$ is the electrostatic potential at the point $\mathbf{r}_{1}$ (the position of the donor) due to the charge density $-e \psi_{g}^{A *}\left(\mathbf{r}_{2}\right) \psi_{e}^{A}\left(\mathbf{r}_{2}\right)$. We shall think of the acceptor as having periodicity, as a result of which the states are characterised by the wave vector $\mathbf{k}$. As a result of energy transfer from the donor, an electron in an energy level with wave vector $\mathbf{k}_{i}$ is excited to a level with wave vector $\mathbf{k}_{f}$. For the transfer of an energy $\hbar \Omega$ from the donor to the acceptor, the rate is given by:

$$
k(\hbar \Omega)=\frac{2 \pi}{\hbar} \sum_{\mathbf{k}_{i}} \sum_{\mathbf{k}_{f}}\left|U_{\mathbf{k}_{i}, \mathbf{k}_{f}}\right|^{2} \delta\left(E_{\mathbf{k}_{f}}-E_{\mathbf{k}_{i}}-\hbar \Omega\right) .
$$

We define $\mathbf{k}_{f}=\mathbf{k}_{i}+\mathbf{q}$, where $\mathbf{q} \hbar$ is the momentum transferred to the acceptor. The rate can therefore be written as:

$$
k(\hbar \Omega)=\frac{2 \pi}{\hbar} \sum_{\mathbf{k}_{i}} \sum_{\mathbf{q}}\left|U_{\mathbf{k}_{i}, \mathbf{q}}\right|^{2} \delta\left(E_{\mathbf{k}_{i}+\mathbf{q}}-E_{\mathbf{k}_{i}}-\hbar \Omega\right) .
$$

The transition density given by $-e \psi_{g}^{A *}\left(\mathbf{r}_{2}\right)$ $\psi_{e}^{A}\left(\mathbf{r}_{2}\right)=-e \psi_{\mathbf{k}_{i}}^{A *}\left(\mathbf{r}_{2}\right) \psi_{\mathbf{k}_{i}+\mathbf{q}}^{A}\left(\mathbf{r}_{2}\right)$ then has a periodicity with wave vector q. If one adopts the simplest possible approximation, the electrostatic potential at a point outside such a charge distribution is a function only of $\mathbf{q}$ i.e. $U_{\mathbf{k}_{i}, \mathbf{q}}=U(\mathbf{q})$ (this is not true for graphene and carbon nanotubes). Therefore, the rate can be written as

$$
k(\hbar \Omega)=\frac{2 \pi}{\hbar} \sum_{\mathbf{q}}|U(\mathbf{q})|^{2} F(\mathbf{q}),
$$

where

$$
F(\mathbf{q})=\sum_{\mathbf{k}_{i}} \delta\left(E_{\mathbf{k}_{i}+\mathbf{q}}-E_{\mathbf{k}_{i}}-\hbar \Omega\right)
$$

The distance dependence of the rate of energy transfer is therefore governed by the functional forms of the interaction energy, $U(\mathbf{q})$ and $F(\mathbf{q})$. We use the general formalism given above to understand the process of energy transfer from a localized donor to the following energy acceptors: graphene, a twodimensional semiconducting sheet and the case of such a semiconducting sheet rolled to obtain a nanotube.

\section{Resonance energy transfer from a dye to graphene}

We now consider the process of energy transfer from a fluorescent dye to graphene (see figure 1). ${ }^{6,7}$ We use the tight-binding model for graphene. ${ }^{8}$ The wave functions for graphene are

$$
\psi_{\mathbf{k}}^{ \pm}(\mathbf{r})=\frac{1}{\sqrt{2 N}}\left[\begin{array}{l}
\sum_{A} e^{i \mathbf{k} \cdot \mathbf{s}_{A}} \chi_{A}\left(\mathbf{r}-\mathbf{s}_{A}\right) \\
\pm e^{-i \delta_{\mathbf{k}}} \sum_{B} e^{i \mathbf{k} \cdot \mathbf{s}_{B}} \chi_{B}\left(\mathbf{r}-\mathbf{s}_{B}\right)
\end{array}\right],
$$

where the + sign holds for the valence band ( $\pi$ band) and the - sign holds for the conduction band $\left(\pi^{*}\right.$ band). $A$ and $B$ refer to the two carbon atoms of the unit cell of graphene. $N$ is the number of atoms of each type in the lattice. $\chi \mathrm{s}$ are the $2 p_{z}$ atomic orbital wave functions of the carbon atoms of the lattice. The phase factor $\delta_{k}$ is defined by the following relation:

$$
e^{i \delta_{\mathbf{k}}}=-\frac{H_{A B}(\mathbf{k})}{\left|H_{A B}(\mathbf{k})\right|},
$$


where

$$
H_{A B}(\mathbf{k})=-t\left[e^{i k_{x} a / \sqrt{3}}+2 e^{-i k_{x} a / 2 \sqrt{3}} \cos \left(k_{y} a / 2\right)\right] .
$$

In the above equation, $t$ is the hopping integral and $a=l_{C-C} \sqrt{3}$. The energies of the bands are given by

$$
\begin{aligned}
E_{\mathbf{k}}^{ \pm}= & \pm t\left[1+4 \cos \left(k_{y} a / 2\right) \cos \left(k_{x} \sqrt{3} a / 2\right)\right. \\
& \left.+4 \cos ^{2}\left(k_{y} a / 2\right)\right]^{1 / 2}
\end{aligned}
$$

where the + sign is for the conduction band and the - sign is for the valence band. The valence and the conduction bands meet at the K-points. Near the Kpoints, ${ }^{9}$ the energy dispersion becomes

$$
E_{\mathbf{k}}^{ \pm}= \pm t \frac{\sqrt{3} a}{2} k= \pm v_{f} k
$$

where $k=\sqrt{k_{x}^{2}+k_{y}^{2}}$.

When the donor, a fluorescent dye molecule is excited, an electron from an occupied orbital is excited to an unoccupied orbital. We consider the

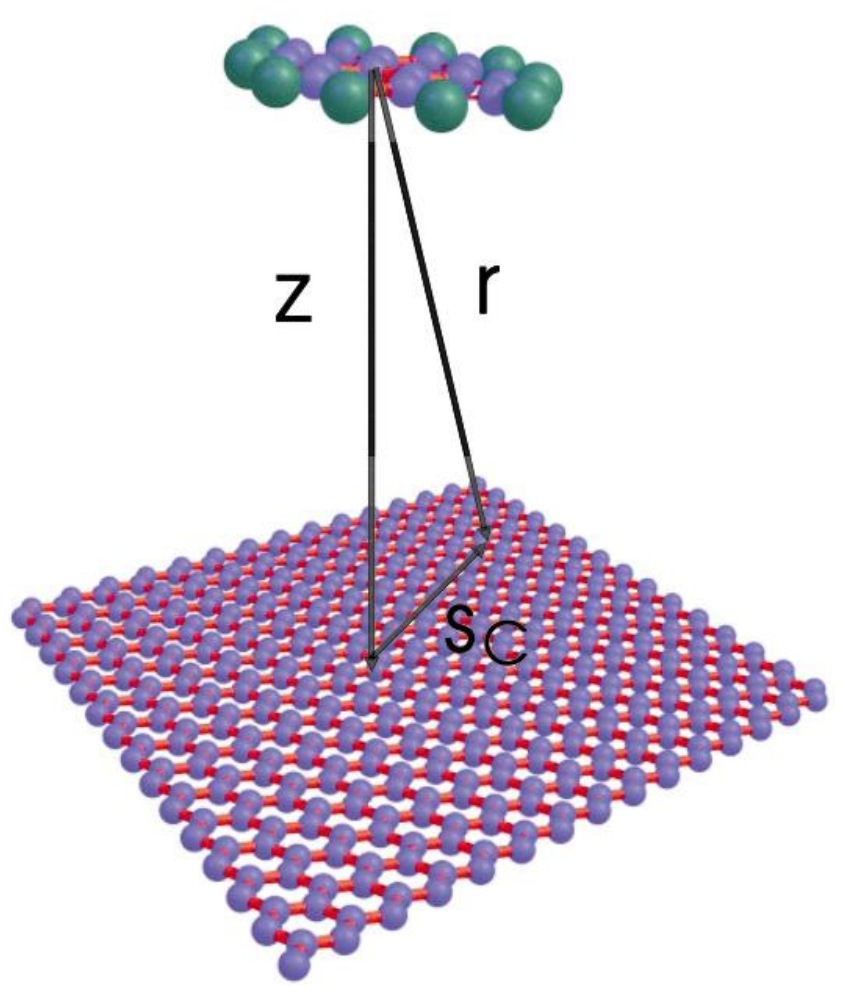

Figure 1. A schematic of the graphene lattice and the donor dye. The position vectors involved in the analysis are also shown. transition dipole moment of the dye, $\boldsymbol{\mu}_{e g}^{D}=\mu_{x} \hat{\mathbf{i}}+\mu_{y} \hat{\mathbf{j}}+\mu_{z} \hat{\mathbf{k}}$ to be interacting with the electrons of graphene. As a result of such an interaction, the dye returns back to its ground state and an electron in graphene is excited. Excitation of an electron from $\psi_{\mathbf{k}_{i}}^{+}(\mathbf{r})$ to $\psi_{\mathbf{k}_{i}+\mathbf{q}}^{-}(\mathbf{r})$ leads to a transition charge density given by

$$
\begin{aligned}
\rho(\mathbf{r})= & -e \psi_{\mathbf{k}_{i}+\mathbf{q}}^{-*}(\mathbf{r}) \psi_{\mathbf{k}_{i}}^{+}(\mathbf{r}) \\
\simeq & \frac{e}{2 N}\left[e^{i\left(\delta_{\mathbf{k}_{i}+\mathbf{q}^{-}}-\delta_{\mathbf{k}_{i}}\right)}-1\right] \\
& \sum_{C} e^{-i \mathbf{q} \cdot \mathbf{s}_{C}} \chi_{C}\left(\mathbf{r}-\mathbf{s}_{C}\right) \chi_{C}^{*}\left(\mathbf{r}-\mathbf{s}_{C}\right),
\end{aligned}
$$

where the summation is over carbon atoms of either type in the lattice. Further, we have neglected the product of $\chi$ s which belong to different atoms of the lattice, as they are negligible. The electrostatic potential due to such a transition density is given by

$$
\Phi(\mathbf{r})=\frac{1}{4 \pi \varepsilon} \int \mathrm{d} \mathbf{r}_{2} \frac{\rho\left(\mathbf{r}_{2}\right)}{\left|\mathbf{r}-\mathbf{r}_{2}\right|}
$$

Since the density $\chi_{C}\left(\mathbf{r}_{2}-\mathbf{s}_{C}\right) \chi_{C}^{*}\left(\mathbf{r}_{2}-\mathbf{s}_{C}\right)$ is localized near the $C^{\text {th }}$ atom, we can use the multipole expansion to calculate its electrostatic potential at the point $\mathbf{r}$. The lowest order term is the monopole term leading to

$$
\Phi(\mathbf{r})=\frac{e}{8 \pi \varepsilon N}\left[e^{i\left(\delta_{\mathbf{k}_{i}+\mathbf{q}}-\delta_{\mathbf{k}_{i}}\right)}-1\right] \sum_{C} \frac{e^{-i \mathbf{q} \mathbf{s}_{C}}}{\left|\mathbf{r}-\mathbf{s}_{C}\right|}
$$

For small values of $q(=|\mathbf{q}|)$, the sum in the above equation may be replaced by an integral so that

$$
\Phi(\mathbf{r})=\frac{e}{8 \pi \varepsilon N A_{u}}\left[e^{i\left(\delta_{\left.\mathbf{k}_{i}+\mathbf{q}^{-} \hat{\mathbf{k}}_{i}\right)}\right)}-1\right] \int \mathrm{d} \mathbf{s}_{C} \frac{e^{-i \mathbf{q} \mathbf{s}_{C}}}{\left|\mathbf{r}-\mathbf{s}_{C}\right|}
$$

where $A_{u}$ is the area of the unit cell of graphene. The two-dimensional integral written above can be evaluated to get

$$
\Phi(\mathbf{r})=\frac{e}{4 \varepsilon A}\left[e^{i\left(\delta_{\mathbf{k}_{i}+\mathbf{q}}-\delta_{\mathbf{k}_{i}}\right)}-1\right] \frac{e^{-q z} e^{-i \mathbf{q} \mathbf{X}}}{q},
$$

where we have used $\mathbf{r}=(\mathbf{X}, z)$, with $\mathbf{X}$ being parallel to the plane of graphene. $A$ is the area of the 
graphene lattice. We evaluate the interaction energy using (7) and find it to be

$$
U=\frac{e}{4 \varepsilon A}\left[1-e^{i\left(\delta_{\mathbf{k}_{i}+\mathbf{q}}-\delta_{\mathbf{k}_{i}}\right)}\right] \boldsymbol{\mu}_{e g}^{D} \cdot(i \hat{\mathbf{q}}+\hat{\mathbf{k}}) e^{-q z} e^{-i \mathbf{q} \mathbf{X}},
$$

where $\hat{\mathbf{q}}=\mathbf{q} / q$ is the unit vector in the direction of $\mathbf{q}$ and $\hat{\mathbf{k}}$ is the unit vector in the $z$ direction. The square of the interaction energy is

$$
\begin{gathered}
|U|^{2}=\frac{e^{2}}{8 \varepsilon^{2} A^{2}}\left[1-\cos \left(\varphi_{\mathbf{k}_{i}+\mathbf{q}}-\varphi_{\mathbf{k}_{i}}\right)\right] \\
\left|\boldsymbol{\mu}_{e g}^{D} \cdot(i \hat{\mathbf{q}}+\hat{\mathbf{k}})\right|^{2} e^{-2 q z} .
\end{gathered}
$$

We now use (9) to obtain the following expression for the rate of transfer:

$$
k(\hbar \Omega)=\frac{\pi e^{2}}{4 \hbar \varepsilon^{2} A^{2}} \sum_{\mathbf{q}}\left|\boldsymbol{\mu}_{e g}^{D} \cdot(i \hat{\mathbf{q}}+\hat{\mathbf{k}})\right|^{2} e^{-2 q z} G(\mathbf{q}),
$$

where

$$
G(\mathbf{q})=\sum_{k_{i}}\left[1-\cos \left(\varphi_{\mathbf{k}_{i}+\mathbf{q}}-\varphi_{\mathbf{k}_{i}}\right)\right] \delta\left(E_{\mathbf{k}_{i}+\mathbf{q}}-E_{\mathbf{k}_{i}}-\hbar \Omega\right) .
$$

Note that the extra term in $\mathrm{G}(\mathbf{q})$ compared to $F(\mathbf{q})$ defined in (11) is due to the phase factor in the wave functions of graphene. We first evaluate $G(\mathbf{q})$. We now use (16) for the energy levels of graphene, replace the sum over $\mathbf{k}_{i}$ by an integral to get

$$
\begin{aligned}
G(\mathbf{q})= & \frac{A}{4 \pi^{2}} \int_{0}^{\infty} \mathrm{d} k_{i} k_{i} \int_{0}^{2 \pi} \mathrm{d} \psi\left[1-\frac{k_{i}+q \cos \psi}{\sqrt{k_{i}^{2}+q^{2}+2 k_{i} q \cos \psi}}\right] \\
& \times \delta\left[v_{f}\left(k_{i}+\sqrt{k_{i}^{2}+q^{2}+2 k_{i} q \cos \psi}\right)-\hbar \Omega\right] .
\end{aligned}
$$

The above integral can be evaluated exactly ${ }^{10}$ to obtain

$$
G(\mathbf{q})=\frac{A}{8 \pi} \frac{q^{2} \Theta\left(\hbar \Omega-q v_{f}\right)}{\sqrt{(\hbar \Omega)^{2}-q^{2} v_{f}^{2}}}
$$

We now substitute the above result into (24), replace the sum over $\mathbf{q}$ by an integral to get

$$
\begin{aligned}
k(\hbar \Omega) & =\frac{e^{2}}{64 \pi h \varepsilon^{2}} \int_{0}^{\infty} d q q \int_{0}^{2 \pi} d \theta\left[\mu_{z}^{2}\right. \\
& \left.+\left(\mu_{x} \cos \theta+\mu_{y} \sin \theta\right)^{2}\right] e^{-2 q z} \frac{\theta\left(\hbar \Omega-q v_{f}\right) q^{2}}{\sqrt{(\hbar \Omega)^{2}-q^{2} v_{f}^{2}}}
\end{aligned}
$$

The integral over $\theta$ can be easily performed to get

$$
\begin{aligned}
& k(\hbar \Omega)=\frac{e^{2}}{64 h \varepsilon^{2}}\left(\mu_{x}^{2}+\mu_{y}^{2}+2 \mu_{z}^{2}\right) \\
& \int_{0}^{\hbar \Omega / v_{f}} \mathrm{~d} q \frac{e^{-2 q z} q^{3}}{\sqrt{(\hbar \Omega)^{2}-q^{2} v_{f}^{2}}}
\end{aligned}
$$

For large values of $z$, only values of $q \simeq 1 /(2 z)$ contribute to the above integral. In such a case, we can neglect $q^{2} v_{f}^{2}$ in comparison with $(\hbar \Omega)^{2}$, and extend the upper limit of the integral in (29) to infinity, to get

$$
k(\hbar \Omega)=\frac{3 \pi e^{2}}{256 \Omega h^{2} \varepsilon^{2}} \frac{\left(\mu_{x}^{2}+\mu_{y}^{2}+2 \mu_{z}^{2}\right)}{z^{4}} .
$$

The above expression for rate can be rewritten as

$$
k(\hbar \Omega)=\frac{3 \pi e^{2}}{256 \Omega h^{2} \varepsilon^{2}} \frac{\left(\mu_{e g}^{2} \sin ^{2} \theta+2 \mu_{e g}^{2} \cos ^{2} \theta\right)}{z^{4}},
$$

where $\left|\boldsymbol{\mu}_{e g}^{D}\right|=\mu_{e g}$ and $\theta$ is the angle that $\boldsymbol{\mu}_{e g}^{D}$ makes with the $z$-axis. We now perform an averaging over all possible orientations of the donor transition dipole moment to get

$$
\langle k(\hbar \Omega)\rangle=\frac{\pi e^{2}}{64 \Omega h^{2} \varepsilon^{2}} \frac{\mu_{e g}^{2}}{z^{4}} .
$$

Therefore, the rate of energy transfer has a power law dependence $\left[(\text { distance })^{-4}\right]$ for large values of $z$.

\section{Resonance energy transfer from a dye to a two-dimensional semiconducting sheet}

We now consider the process of energy transfer from a dye molecule to the electrons confined to a two-dimensional semiconducting sheet. We assume 
that there is a band gap $\varepsilon_{g}$ and the energy levels above and below the gap are given by

$$
E_{\mathbf{k}}^{ \pm}= \pm\left(\frac{\varepsilon_{g}}{2}+\frac{\hbar^{2} \mathbf{k}^{2}}{2 m^{*}}\right)
$$

The + sign is for the conduction band and the - sign is for the valence band. $\mathbf{k}$ is a two-dimensional wave vector. We have assumed the effective mass $m^{*}$ to be the same for states above and below the band gap. The corresponding wave functions are

$$
\psi_{\mathbf{k}}^{ \pm}(\mathbf{r})=\frac{1}{\sqrt{N}} \sum_{i=1}^{N} e^{i \mathbf{k} \cdot \mathbf{R}_{\mathbf{i}}} \chi^{ \pm}\left(\mathbf{r}-\mathbf{R}_{i}\right),
$$

where the + sign is for the conduction band and the - sign is for the valence band. $\mathbf{R}_{i}$ denotes the position of the $i$ th atom in the sheet. $N$ is the total number of atoms of the sheet. $\chi^{+}\left(\mathbf{r}-\mathbf{R}_{l}\right)$ and $\chi^{-}\left(\mathbf{r}-\mathbf{R}_{i}\right)$ denote the localized orbitals on the $i$ th atom that contribute to the conduction and the valence bands respectively. With the above, excitation of an electron from $\psi_{\mathbf{k}_{i}}^{-}(\mathbf{r})$ to $\psi_{\mathbf{k}_{i}+\mathbf{q}}^{+}(\mathbf{r})$ leads to a transition charge density given by

$$
\begin{aligned}
\rho(\mathbf{r})= & -e \psi_{\mathbf{k}_{i}+\mathbf{q}}^{+*}(\mathbf{r}) \psi_{\mathbf{k}_{i}}^{-}(\mathbf{r}) \\
& \simeq \frac{-e}{N} \sum_{i=1}^{N} e^{-i q \cdot \mathbf{R}_{i}} \chi^{-}\left(\mathbf{r}-\mathbf{R}_{i}\right) \chi^{+*}\left(\mathbf{r}-\mathbf{R}_{i}\right),
\end{aligned}
$$

where we have neglected the product of $\chi$ s which belong to different atoms of the lattice, as they are negligible. The electrostatic potential due to such a transition density is given by

$$
\Phi(\mathbf{r})=\frac{1}{4 \pi \varepsilon} \int \mathrm{d} \mathbf{r}_{2} \frac{\rho\left(\mathbf{r}_{2}\right)}{\left|\mathbf{r}-\mathbf{r}_{2}\right|} .
$$

Since the density $\chi^{-}\left(\mathbf{r}_{2}-\mathbf{R}_{i}\right) \chi^{+*}\left(\mathbf{r}_{2}-\mathbf{R}_{i}\right)$ is localized near the $i$ th atom, we can use the multipole expansion to calculate its electrostatic potential at the point $\mathbf{r}$. The monopole term of the multipole expansion gives zero since the two orbitals $\chi^{-}$and $\chi^{+}$ are orthogonal. Therefore, the lowest order non-zero term is dipolar in nature and one gets

$$
\Phi(\mathbf{r})=\frac{1}{4 \pi \varepsilon N} \boldsymbol{\mu}_{ \pm} \cdot \nabla_{\mathbf{r}} \sum_{i=1}^{N} \frac{e^{-i \mathbf{q} \cdot \mathbf{R}_{i}}}{\left|\mathbf{r}-\mathbf{R}_{i}\right|}
$$

where

$$
\boldsymbol{\mu}_{ \pm}=-e \int d \mathbf{r}_{2} \chi^{+*}\left(\mathbf{r}_{2}-\mathbf{R}_{i}\right)\left(\mathbf{r}_{2}-\mathbf{R}_{i}\right) \chi^{-}\left(\mathbf{r}_{2}-\mathbf{R}_{i}\right)
$$

is the transition dipole for the $\chi^{-} \rightarrow \chi^{+}$transition. For small values of $q(=|\mathbf{q}|)$, the sum in the above equation may be replaced by an integral so that

$$
\Phi(\mathbf{r})=\frac{1}{4 \pi \varepsilon A_{u} N} \boldsymbol{\mu}_{ \pm} \cdot \nabla_{\mathbf{r}} \int \mathrm{d} \mathbf{R}_{i} \frac{e^{-i \mathbf{q} \cdot \mathbf{R}_{i}}}{\left|\mathbf{r}-\mathbf{R}_{i}\right|},
$$

where $A_{u}$ is the area of the unit cell of the twodimensional lattice. We consider that the unit cell of the lattice contains one atom such that $A=N A_{u}$, where $A$ is the area of the sheet. The twodimensional integral written above can be evaluated to get

$$
\Phi(\mathbf{r})=\frac{1}{2 \varepsilon A} \boldsymbol{\mu}_{ \pm} \cdot\left[\nabla_{\mathbf{r}}\left(\frac{e^{-q z} e^{-i \mathbf{q} \cdot \mathbf{x}}}{q}\right)\right],
$$

where we have used $\mathbf{r}=(\mathbf{X}, z)$, with $\mathbf{X}$ being parallel to the plane of the sheet. The above equation can be simplified to obtain

$$
\Phi(\mathbf{r})=\frac{-1}{2 \varepsilon A} \boldsymbol{\mu}_{ \pm} \cdot(i \hat{\mathbf{q}}+\hat{\mathbf{k}}) e^{-q z} e^{-i \mathbf{q} \cdot \mathbf{x}} .
$$

We now use (7) to get

$$
U=\frac{1}{2 \varepsilon A} \boldsymbol{\mu}_{ \pm} \cdot(i \hat{\mathbf{q}}+\hat{\mathbf{k}}) \boldsymbol{\mu}_{e g}^{D} \cdot(i \hat{\mathbf{q}}+\hat{\mathbf{k}}) q e^{-q z} e^{-i \mathbf{q} \cdot \mathbf{x}}
$$

where $\boldsymbol{\mu}_{e g}^{D}$ is the transition dipole moment of the dye given by $\boldsymbol{\mu}_{\text {eg }}^{D}=\mu_{x}^{D} \hat{\mathbf{i}}+\mu_{y}^{D} \hat{\mathbf{j}}+\mu_{z}^{D} \hat{\mathbf{k}}$. Note that $U$ is a function only of $\mathbf{q}$. Therefore, we substitute (41) into (10) to get the rate of transfer given by

$$
\begin{aligned}
k(\hbar \Omega)=\frac{\pi}{2 \hbar \varepsilon^{2} A^{2}} & \sum_{\mathbf{q}}\left|\boldsymbol{\mu}_{ \pm} \cdot(i \hat{\mathbf{q}}+\hat{\mathbf{k}})\right|^{2} \\
& \left|\boldsymbol{\mu}_{e g}^{D} \cdot(i \hat{\mathbf{q}}+\hat{\mathbf{k}})\right|^{2} q^{2} e^{-2 q z} F(\mathbf{q}) .
\end{aligned}
$$

We now evaluate $F(\mathbf{q})$ using (33) and (11) to get

$$
F(\mathbf{q})=\frac{m^{*} A}{2 \pi \hbar^{2}} \Theta\left[\frac{2 m^{*}}{\hbar^{2}}\left(\hbar \Omega-\varepsilon_{g}\right)-q^{2}\right] .
$$


We now substitute this back into (42) and replace the sum over $\mathbf{q}$ by an integral to obtain the following expression for the rate:

$$
\begin{aligned}
k(\hbar \Omega)= & \frac{m^{*}}{16 \pi^{2} \varepsilon^{2} \hbar^{3}} \int_{0}^{\infty} \mathrm{d} q q^{3} e^{-2 q z} \Theta\left[\frac{2 m^{*}}{\hbar^{2}}\left(\hbar \Omega-\varepsilon_{g}\right)-q^{2}\right] \\
& \times \int_{0}^{2 \pi} \mathrm{d} \theta\left|\boldsymbol{\mu}_{ \pm} \cdot(i \hat{\mathbf{q}}+\hat{\mathbf{k}})\right|^{2}\left|\boldsymbol{\mu}_{e g}^{D} \cdot(i \hat{\mathbf{q}}+\hat{\mathbf{k}})\right|^{2}
\end{aligned}
$$

The two-dimensional vector $\mathbf{q}$ is expressed in its polar coordinates $q$ and $\theta$. For large $z$, only small values of $q$ are important. Then,

$$
\Theta\left[\frac{2 m^{*}}{\hbar^{2}}\left(\hbar \Omega-\varepsilon_{g}\right)-q^{2}\right] \simeq \Theta\left[\frac{2 m^{*}}{\hbar^{2}}\left(\hbar \Omega-\varepsilon_{g}\right)\right]=1,
$$

if $\hbar \Omega>\varepsilon_{g}$. For such a situation, the integral over $q$ can be exactly evaluated to obtain the following expression for the rate

$$
\begin{aligned}
k(\hbar \Omega)= & \frac{3 m^{*}}{128 \pi^{2} \varepsilon^{2} \hbar^{3} z^{4}} \\
& \int_{0}^{2 \pi} \mathrm{d} \theta\left|\boldsymbol{\mu}_{ \pm} \cdot(i \hat{\mathbf{q}}+\hat{\mathbf{k}})\right|^{2}\left|\boldsymbol{\mu}_{e g}^{D} \cdot(i \hat{\mathbf{q}}+\hat{\mathbf{k}})\right|^{2} .
\end{aligned}
$$

The integral over $\theta$ can now be performed to get an expression involving the components of the dipole moments, $\boldsymbol{\mu}_{ \pm}$and $\boldsymbol{\mu}_{e g}^{D}$. We then perform an averaging over all possible orientations of the donor dipole, $\boldsymbol{\mu}_{e g}^{D}$ leading to the following expression for the rate:

$$
\begin{aligned}
\langle k(\hbar \Omega)\rangle= & \frac{m^{*}}{64 \pi \varepsilon^{2} \hbar^{3} z^{4}} \\
& \mu_{e g}^{2}\left(\mu_{x}^{ \pm^{2}}+\mu_{y}^{ \pm^{2}}+2 \mu_{z}^{ \pm^{2}}\right) \text { if } \hbar \Omega>\varepsilon_{g} \\
& =0 \text { if } \hbar \Omega<\varepsilon_{g}
\end{aligned}
$$

where we have used $\left|\boldsymbol{\mu}_{e g}^{D}\right|=\mu_{e g} \quad$ and $\boldsymbol{\mu}_{ \pm}=\mu_{x}^{ \pm} \hat{\mathbf{i}}+\mu_{y}^{ \pm} \hat{\mathbf{j}}+\mu_{z}^{ \pm} \hat{\mathbf{k}}$. The rate of transfer in this case too has a (distance) ${ }^{-4}$ dependence.

\section{Resonance energy transfer from a dye to a long tubule}

We now consider the process of energy transfer from a dye molecule to the electrons confined to a very long tubule of radius $a$ (which is of nanodimensions) and length $L(\rightarrow \infty)$ (see figure 2). We adopt the simplest possible description for the electrons of the tubule. We assume that we have a semiconducting nanotube, having a structure similar to the boron-nitride nanotube, with a band gap $\varepsilon_{g}$ (see figure 3). ${ }^{11}$ The case of carbon nanotube is interesting and is discussed in a separate publication. ${ }^{12}$ The states above and below the band gap may be characterized by the momentum parallel to the axis of the tube, which we denote by $k$ and the angular momentum about the tube axis, which is quantized (angular momentum being equal to $m \hbar$, with $m=0, \pm 1, \pm 2, \ldots){ }^{13}$ We take the electron wave functions to be

$$
\psi_{k, m}^{ \pm}(\rho, \phi, z)=\frac{1}{\sqrt{N}} \sum_{i=1}^{N} e^{i k z_{i}+i m \varphi_{i}} \chi^{ \pm}\left(\mathbf{r}-\mathbf{R}_{i}\right)
$$

The superscripts \pm in $\psi_{k, m}^{ \pm}(\rho, \phi, z)$ denote states above $(+)$ or below $(-)$ the gap. $\chi^{+}\left(\mathbf{r}-\mathbf{R}_{i}\right)$ and $\chi^{-}$ $\left(\mathbf{r}-\mathbf{R}_{i}\right)$ denote the localized orbitals on the $i$ th lattice site that contribute to the conduction and the valence bands respectively. $N$ is the number of lattice sites of the tubule. We take the energies of the states to be given by ${ }^{14}$

$$
\varepsilon_{k, m}^{ \pm}= \pm\left(\frac{\varepsilon_{g}}{2}+\frac{\hbar^{2} k^{2}}{2 m^{*}}+\frac{\hbar^{2} m^{2}}{2 m^{*} a^{2}}\right) \text {, }
$$

where $m^{*}$ is the effective mass. With the above, excitation of an electron from $\psi_{k_{i}, m_{i}}^{-}(\mathbf{r})$ to $\psi_{k_{f}, m_{f}}^{+}(\mathbf{r})$ leads to a transition charge density given by



Figure 2. A schematic of the transition dipole moment of the donor dye and the tube. 


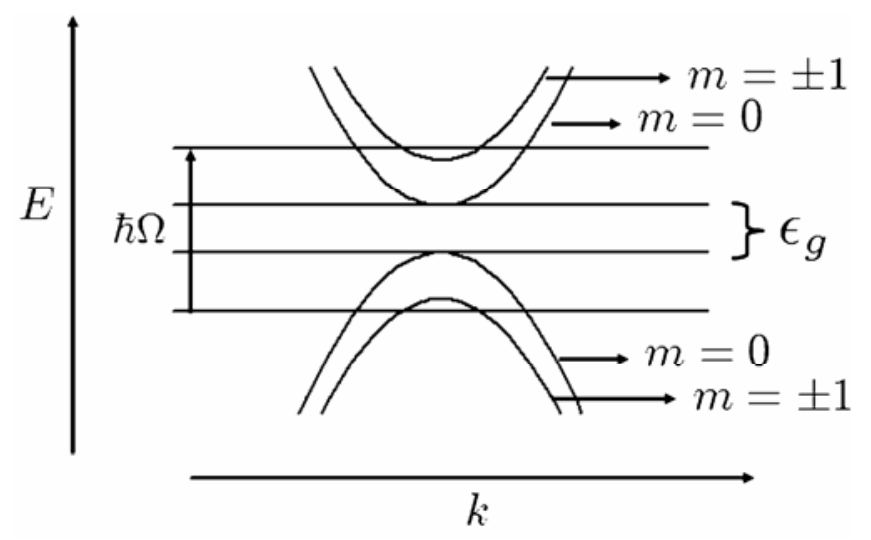

Figure 3. A schematic of the one-dimensional energy bands of the nanotube showing the quantum numbers along the equatorial direction, the energy gap and the amount of energy transferred.

$$
\begin{aligned}
\rho(\mathbf{r})= & -e \psi_{k_{f}, m_{f}}^{+*}(\mathbf{r}) \psi_{k_{i}, m_{i}}^{-}(\mathbf{r}) \\
& \simeq \frac{-e}{N} \sum_{i=1}^{N} e^{-i q z_{i}} e^{-i m_{q} \varphi_{i}} \chi^{-}\left(\mathbf{r}-\mathbf{R}_{i}\right) \chi^{+*}\left(\mathbf{r}-\mathbf{R}_{i}\right)
\end{aligned}
$$

where we have put $k_{f}=k_{i}+q, \quad m_{f}=m_{i}+m_{q}$ and neglected the product of $\chi \mathrm{s}$ which belong to different sites of the lattice, as they are negligible. $\left(a, \varphi_{i}, z_{i}\right)$ are the cylindrical polar coordinates of $\mathbf{R}_{i}$. The electrostatic potential due to such a transition density is given by

$$
\Phi(\mathbf{r})=\frac{1}{4 \pi \varepsilon} \int \mathrm{d} \mathbf{r}_{2} \frac{\rho\left(\mathbf{r}_{2}\right)}{\left|\mathbf{r}-\mathbf{r}_{2}\right|}
$$

Since the density $\chi^{-}\left(\mathbf{r}_{2}-\mathbf{R}_{i}\right) \chi^{+*}\left(\mathbf{r}_{2}-\mathbf{R}_{i}\right)$ is localized near the $i$ th atom, one can write the potential as

$$
\Phi(\mathbf{r})=\frac{1}{4 \pi \varepsilon N} \boldsymbol{\mu}_{ \pm} \cdot \nabla_{\mathbf{r}} \sum_{i=1}^{N} \frac{e^{-i q z_{i}} e^{-i m_{q} \varphi_{i}}}{\left|\mathbf{r}-\mathbf{R}_{i}\right|}
$$

where $\boldsymbol{\mu}_{ \pm}=-e \int d \mathbf{r}_{2} \chi^{+*}\left(\mathbf{r}_{2}-\mathbf{R}_{i}\right)\left(\mathbf{r}_{2}-\mathbf{R}_{i}\right) \chi^{-}\left(\mathbf{r}_{2}-\mathbf{R}_{i}\right)$ is the transition dipole for the $\chi^{-} \rightarrow \chi^{+}$transition. For small values of $q$, the sum in the above equation may be replaced by an integral so that

$$
\Phi(\mathbf{r})=\frac{1}{4 \pi \varepsilon A_{u} N} \boldsymbol{\mu}_{ \pm} \cdot \nabla_{\mathbf{r}} \int \mathrm{d} \mathbf{R}_{i} \frac{e^{-i q z_{i}} e^{-i m_{q} \varphi_{i}}}{\left|\mathbf{r}-\mathbf{R}_{i}\right|}
$$

where $A_{u}$ is the surface area of the unit cell of the cylindrical surface. We consider that the unit cell of the lattice contains one atom leading to $A=N A_{u}$, where $A$ is the surface area of the long cylinder. We now use the following multipole expansion for the $1 /\left|\mathbf{r}-\mathbf{R}_{i}\right|$ term in cylindrical coordinates: ${ }^{15}$

$$
\begin{gathered}
\frac{1}{\left|\mathbf{r}-\mathbf{R}_{i}\right|}=\frac{1}{\pi} \sum_{m^{\prime}=-\infty}^{\infty} \int_{-\infty}^{\infty} \mathrm{d} k^{\prime} e^{i m^{\prime}\left(\varphi-\varphi_{i}\right)} e^{i k^{\prime}\left(z-z_{i}\right)} \\
I_{m^{\prime}}\left(\left|k^{\prime}\right| a\right) K_{m^{\prime}}\left(\left|k^{\prime}\right| \mathrm{d} R\right),
\end{gathered}
$$

where we have used $\mathbf{r}=\mathrm{d} \cos \varphi \hat{\mathbf{i}}+\mathrm{d} \sin \varphi \hat{\mathbf{j}}+z \hat{\mathbf{k}}$ and $\mathbf{R}_{i}=a \cos \varphi_{i} \hat{\mathbf{i}}+a \sin \varphi_{i} \hat{\mathbf{j}}+z_{i} \hat{\mathbf{k}}$. On substituting this back into (52) and evaluating the subsequent integrals, one gets

$$
\Phi(\mathbf{r})=\frac{a}{\varepsilon A} I_{\left|m_{q}\right|}(|q| a) \boldsymbol{\mu}_{ \pm} \cdot \nabla_{\mathbf{r}}\left[e^{-i q z} e^{-i m_{q} \varphi} K_{\left|m_{q}\right|}(|q| d)\right] .
$$

We now use $\boldsymbol{\mu}_{ \pm}=\mu_{\rho}^{ \pm} \hat{\boldsymbol{\rho}}+\mu_{\varphi}^{ \pm} \hat{\boldsymbol{\varphi}}+\mu_{z}^{ \pm} \hat{\mathbf{z}}$ to obtain

$$
\begin{gathered}
\Phi(\mathbf{r})=\frac{a}{\varepsilon A} I_{\left|m_{q}\right|}(|q| a) e^{-i m_{q} \varphi} e^{-i q z} \\
{\left[\begin{array}{l}
-\frac{\mu_{\rho}^{ \pm}|q|}{2}\left(K_{\left|m_{q}\right|-1}(|q| d)+K_{\left|m_{q}\right|+1}(|q| d)\right) \\
-i\left(\frac{m_{q} \mu_{\varphi}^{ \pm}}{d}+q \mu_{z}^{ \pm}\right) K_{\left|m_{q}\right|}(|q| d)
\end{array}\right] .}
\end{gathered}
$$

We now evaluate the interaction energy using (7) and it is given by

$$
U=\frac{a}{\varepsilon A} I_{\left|m_{q}\right|}(|q| a) e^{-i m_{q} \varphi} e^{-i q z}
$$

$$
\left.\left[\begin{array}{c}
\frac{\partial^{2} K_{\left|m_{q}\right|}(|q| d)}{\partial d^{2}} \mu_{\rho}^{ \pm} \mu_{\rho}^{D}-i \frac{\partial K_{\left|m_{q}\right|}(|q| d)}{\partial d} \\
\left\{\frac{m_{q}}{d}\left(\mu_{\varphi}^{ \pm} \mu_{\rho}^{D}+\mu_{\varphi}^{D} \mu_{\rho}^{ \pm}\right)+q\left(\mu_{z}^{D} \mu_{\rho}^{ \pm}+\mu_{z}^{ \pm} \mu_{\rho}^{D}\right)\right\} \\
+K_{\left|m_{q}\right|}(|q| d)\left\{\frac{i m_{q}}{d^{2}} \mu_{\varphi}^{ \pm} \mu_{\rho}^{D}-\frac{m_{q}^{2}}{d^{2}} \mu_{\varphi}^{ \pm} \mu_{\varphi}^{D}-q^{2} \mu_{z}^{ \pm} \mu_{z}^{D}\right. \\
-\frac{q m_{q}}{d}\left(\mu_{z}^{ \pm} \mu_{\varphi}^{D}+\mu_{\varphi}^{ \pm} \mu_{z}^{D}\right)
\end{array}\right\}\right],
$$


where we have used $\boldsymbol{\mu}_{e g}^{D}=\mu_{\rho}^{D} \hat{\boldsymbol{\rho}}+\mu_{\varphi}^{D} \hat{\varphi}+\mu_{z}^{D} \hat{\mathbf{z}}$. We now evaluate $F(\mathbf{q})$ using (11). We use the energy levels from (48) to obtain the following equation for $F(\mathbf{q})$ :

$$
\begin{aligned}
F(\mathbf{q})= & \frac{L m^{*}}{\pi \hbar^{2}} \sum_{m_{i}} \int_{-\infty}^{\infty} \mathrm{d} k_{i} \\
& \delta\left[\begin{array}{l}
\left(k_{i}+q\right)^{2}+k_{i}^{2}+\frac{\left(m_{i}+m_{q}\right)^{2}+m_{i}^{2}}{a^{2}} \\
-\frac{2 m^{*}\left(\hbar \Omega-\varepsilon_{g}\right)}{\hbar^{2}}
\end{array}\right] .
\end{aligned}
$$

On evaluating the integral, we obtain

$$
F(\mathbf{q})=\frac{L m^{*}}{\pi \hbar^{2}} \sum_{\left\{m_{i}\right\}} \frac{1}{\sqrt{\frac{4 m^{*}\left(\hbar \Omega-\varepsilon_{g}\right)}{\hbar^{2}}}} .
$$

The angular brackets over $m_{i}$ indicate that the summation includes those values of $m_{i}$ for which the quantity within the square root term is positive. For small values of momentum transfer (which determine the long distance behaviour), we can neglect the $q^{2}$ term in comparison with

$$
\frac{4 m^{*}\left(\hbar \Omega-\varepsilon_{g}\right)}{\hbar^{2}}-\frac{2\left\{\left(m_{i}+m_{q}\right)^{2}+m_{i}^{2}\right\}}{a^{2}} .
$$

With this condition,

$$
F(\mathbf{q}) \simeq \frac{L m^{*}}{\pi \hbar^{2}} \sum_{\left\{m_{i}\right\}} \frac{1}{\sqrt{\frac{4 m^{*}\left(\hbar \Omega-\varepsilon_{g}\right)}{\hbar^{2}}}} .
$$

Substituting (59) into (10) and replacing the sum over $\mathbf{q}$ by an integral gives

$$
\begin{aligned}
& k(\hbar \Omega)=\frac{L^{2} m^{*}}{\pi \hbar^{3}} \\
& \left.\sum_{\left\{m_{i}\right\}, m_{q}} \sqrt[1]{\sqrt{\frac{4 m^{*}\left(\hbar \Omega-\varepsilon_{g}\right)}{\hbar^{2}}}}\left|\int_{-\infty}^{\infty} \mathrm{d} q\right| U(\mathbf{q})\right|^{2},
\end{aligned}
$$

where $U(\mathbf{q})$ is given by (56). To evaluate the integral over $q$, we change the variable of integration to $t$ defined by $t=q d$ and subsequently use the asymptotic form of

$$
I_{\left|m_{q}\right|}\left(\frac{|t| a}{d}\right)
$$

given by

$$
I_{\left|m_{q}\right|}\left(\frac{|t| a}{d}\right) \simeq\left(\frac{|t| a}{2 d}\right)^{\left|m_{q}\right|} \frac{1}{\Gamma\left(\left|m_{q}\right|+1\right)} .
$$

The integral over $t$ can now be easily evaluated for various values of $m_{q}$. The leading order term in the expression for the rate corresponds to the case when $m_{q}=0$. We first evaluate the integral over $t$ for such a case and then average over all possible orientations of the donor transition dipole moment to get the following expression for the rate:

$$
\begin{array}{r}
k(\hbar \Omega)=\frac{3 m^{*} \frac{\left(33 \mu_{z}^{ \pm^{2}}+71 \mu_{\rho}^{ \pm^{2}}\right)}{d^{5}}}{8192 \pi \hbar^{3} \varepsilon^{2}} \frac{\mu_{e g}^{2}}{\sum_{\left\{m_{i}\right\}} \sqrt{\sqrt{\frac{m^{*}\left(\hbar \Omega-\varepsilon_{g}\right)}{\hbar^{2}}-\frac{m_{i}^{2}}{a^{2}}}},}
\end{array}
$$

where we have used $\left|\boldsymbol{\mu}_{e g}^{D}\right|=\mu_{e g}$. Therefore, the leading order term in the expression for the rate has a (distance $^{-5}$ dependence.

\section{Discussion}

In the recent times, a variety of materials like polymers, ${ }^{16}$ nanoparticles, ${ }^{17}$ quantum wells, ${ }^{18}$ quantum dots, quantum wires, ${ }^{19}$ metal surfaces ${ }^{20}$ etc. have been used as energy donors/acceptors and they have led to deviations from the $R^{-6}$ dependence. When the energy transfer occurs from a localized excitation on the donor to a localized excitation on the acceptor, the dipolar approximation works and therefore one has an $R^{-6}$ dependence. On the contrary, if the energy transfer process involves an extended electronic system where the excitations are delocalized like in the case of polymers, quantum wires, quantum wells, the dipolar approximation is not valid and one can have deviations from the $R^{-6}$ 
rates. A non- $R^{-6}$ dependence is of great interest due to the need for developing nanoscopic rulers, that can measure distances well beyond $100 \AA$.

Chance et $a l^{21}$ have theoretically analysed the process of decay of an excited molecule fluorescing near a metallic film. They found a $d^{-3}$ dependence of the rate of energy transfer on the distance between the molecule and the metal for very thick films. But, for the case of thin metallic films, they found a $d^{4}$ dependence. Campion et al. ${ }^{22}$ experimentally studied the process of electronic excitation energy transfer from pyrazine to a single crystal metal surface and found a $d^{-3}$ dependence of the rate. A $d^{4}$ dependence has also been observed for the decay of biacetyl above a silver surface. ${ }^{20}$ Further, Persson and Lang ${ }^{23}$ studied the process of energy transfer from a vibrating dipole to the conduction electrons of a metal. They find that the energy transfer to the surface modes of the metal leads to a $d^{-4}$ dependence of the rate on the distance, while the transfer to the bulk modes leads to a $d^{-3}$ dependence. For the case of energy transfer to the surface plasmon modes of a flat metal surface from a proximal dipolar emitter, ${ }^{24}$ quartic distance dependence has been reported. The above dependencies can be easily understood. In FRET, the transfer occurs from a zero-dimensional donor to a zero-dimensional acceptor leading to an $R^{-6}$ dependence. For the case of transfer to the bulk modes of the metal, the acceptor excitations are in three-dimensions. Therefore, one has a $d^{-3}$ dependence. When the transfer occurs to the acceptors where the excitations are confined to two dimensions, one has a $d^{-4}$ dependence.

Lyo $^{25}$ has studied the process of excitonic energy transfer from a narrow quantum wire to a wide quantum wire. The rate of transfer was found to have a $d^{4}$ dependence for intermediate values of $d$ and a $d^{-5}$ dependence in the asymptotic limit for large values of $d$. He has also analysed the case of transfer from a narrow quantum wire to a uniform array of identical parallel wide quantum wires and found a $d^{4}$ dependence in such a case.

Very recently, Govorov et al. ${ }^{19}$ reported a study of the process of excitation energy transfer from a nanoparticle to a nanowire. When the radius of the nanoparticle is small compared to the distance between the nanoparticle and the nanowire, the excitations in nanoparticles can be considered localized and hence dipolar. For large nanoparticlenanowire distances, they find a $d^{-5}$ dependence of the rate on the distance. Since the acceptor excitations are confined to one-dimension, the rate has a $d^{-5}$ dependence.

Energy transfer from an inorganic quantum well to an organic polyfluorene film has been found to occur $^{26}$ due to the Coulombic coupling between the Mott-Wannier excitons on the quantum well to the Frenkel excitons on the polymer. The rate was found to have a $d^{-2}$ dependence. Similar distance dependence has been found earlier for the case of electronic excitation energy transfer from polyfluorene to tetraphenyl porphyrin. ${ }^{16}$ Hill et $a l^{27}$ have also reported a similar distance dependence for the case of energy transfer between two fluorescent polymers that are grown as layered structures. Since the excitations in polymer films and quantum wells are delocalized over two-dimensions, the rate has a $d^{-2}$ dependence.

The process of energy transfer from a quantum well ${ }^{18}$ to a monolayer of semiconductor nanocrystals was reported recently. The experiments revealed very high efficiencies of energy transfer and the process was attractive for use in a variety of technological applications like LEDs, lasers, etc. Kos et $a l^{28}$ have studied this process theoretically assuming that the size of the quantum dot and the width of the quantum well are small in comparison with the distance between them. They considered two types of excited states in the quantum dots as a result of transfer, namely a quasicontinuum of high energy states and discrete low energy states. For the case of transfer to a quasicontinuum of states, they find a $d^{4}$ dependence of the rate, while for excitation to discrete low energy states, they find an exponential dependence. Agranovich et $a l^{29}$ have considered the process of energy transfer from an inorganic quantum well to an organic layer and found an exponential distance dependence.

In all the above cases, either the donor or the acceptor or both were extended systems with delocalized charge densities. Therefore, breakdown of the dipolar approximation to the rate led to deviations from the usual $R^{-6}$ dependence. Strouse et $a l .{ }^{30}$ have studied the process of energy transfer from the dye fluorescein to a $1.4 \mathrm{~nm}$ diameter gold nanoparticle. Double-stranded DNA molecules of various lengths were used to fix the distances between the donor and the acceptor. Surprisingly, though the distances between the donor and the acceptor are much larger than the dimensions of the donor and the acceptor, they find a $d^{4}$ dependence of the rate. They refer to this process as nanoparticle surface energy transfer (NSET) and the range of 
distances that can be measured using NSET is more than double that of the traditional FRET experiments. Therefore, it is interesting to ask why the distance dependence in NSET is $d^{4}$. But, theoretical calculations for such a system considering the transfer to the plasmons and the electron-hole pair excitations of the nanoparticle predominantly find an $R^{-6}$ dependence. ${ }^{31,32}$

\section{Conclusions}

We have studied the distance dependence of the process of resonance energy transfer from a localized donor, a dye molecule to various types of energy acceptors-graphene, a two-dimensional semiconducting sheet and a semiconducting nanotube using simple analytic models. For the case of transfer to graphene and a two-dimensional semiconducting sheet of electronic charge density, we find a

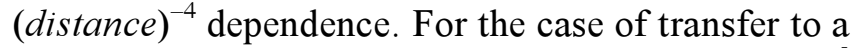
semiconducting nanotube, we find a (distance) ${ }^{-5}$ dependence. We hope that simple analytic models of energy transfer presented here can lead to interesting physical insights into the process of resonance energy transfer.

\section{Acknowledgements}

R S Swathi acknowledges Council of Scientific and Industrial Research (CSIR), India and BristolMyers-Squibb Fellowship for financial support.

\section{References}

1. Lakowicz J R 2006 Principles of fluorescence spectroscopy (New York: Springer)

2. May V and Kuhn O 2000 Charge and energy transfer dynamics in molecular systems (Wiley-VCH)

3. Förster T 1948 Ann. Phys. 255

4. Valeur B 2002 Molecular fluorescence (New York: Wiley-VCH)

5. Stryer L and Haugland R P 1967 Proc. Natl. Acad. Sci. USA 58719
6. Swathi R S and Sebastian K L 2008 J. Chem. Phys. 129054703

7. Swathi R S and Sebastian K L 2009 J. Chem. Phys. 130086101

8. Wallace P R 1947 Phys. Rev. 71622

9. Katsnelson M I 2007 Mater. Today 1020

10. Shung Kenneth W K 1986 Phys. Rev. B34 979

11. Margulis V A, Muyumin E E and Gaiduk E A 2008 Phys. Rev. B77 035425

12. Swathi R S and Sebastian K L (unpublished)

13. Balassis A and Gumbs G 2006 Phys. Rev. B74 045420

14. Gumbs G and Balassis A 2005 Phys. Rev. B71 235410

15. Jackson J D 1975 Classical electrodynamics (New York: Wiley Eastern Limited)

16. Wong K F, Bagchi B and Rossky P J 2004 J. Phys. Chem. A108 5752

17. Sönnichsen C, Reinhard B M, Liphardt J and Alivisatos A P 2005 Nat. Biotechnol. 23741

18. Achermann M, Petruska M A, Kos S, Smith D L, Koleske D D and Klimov V I 2004 Nature 429642

19. Martinez P L H and Govorov A O 2008 Phys. Rev. B78 035314

20. Alivisatos A P, Valdeck D H and Harris C B $1985 \mathrm{~J}$. Chem. Phys. 82541

21. Chance R R, Prock A and Silbey R 1978 Adv. Chem. Phys. 371

22. Campion A, Gallo A R, Harris C B, Robota H J and Whitmore P M 1980 Chem. Phys. Lett. 73447

23. Persson B N J and Lang N D 1982 Phys. Rev. B26 5409

24. Larkin I A, Stockman M I, Achermann M and Klimov V I 2004 Phys. Rev. B69 $121403(\mathrm{R})$

25. Lyo S K 2006 Phys. Rev. B73 205322

26. Itskos G, Heliotis G, Lagoudakis P G, Lupton J, Barradas N P, Alves E, Pereira S, Watson I M, Dawson M D, Feldmann J, Murray R and Bradley D D C 2007 Phys. Rev. B76 035344

27. Hill J, Heriot S Y, Worsfold O, Richardson T H and Fox A M 2004 Phys. Rev. B69 041303 (R)

28. Kos S, Achermann M, Klimov V I and Smith D L 2005 Phys. Rev. B71 205309

29. Agranovich V M, Basko D M, La Rocca G C and Bassani F 1998 J. Phys.: Condens. Matter 109369

30. Yun C S, Javier A, Jennings T, Fisher M, Hira S, Peterson S, Hopkins B, Reich N O and Strouse G F 2005 J. Am. Chem. Soc. 1273115

31. Swathi R S and Sebastian K L 2007 J. Chem. Phys. 126234701

32. Bhowmick S, Saini S, Shenoy V B and Bagchi B 2006 J. Chem. Phys. 125181102 